\title{
Quinoline Based 2-Pyrazoline Derivatives Synthetic and Pharmacological Approach
}

\author{
KUMAR SANJEEV S. LAMANI* and OBLENNAVAR KOTRESH
}

Department of Chemistry, C. S. Bembalagi Arts, S. M. R. Palaresha Science and G. L. Rathi Commerce College, Ramdurg-591123, India

san_lamani09@rediffmail.com

Received 5 March 2013 / Accepted 30 April 2013

\begin{abstract}
A series of 2-pyrazolines has been synthesized by reaction with quinoline and acetyl sydnone derivatives as the starting materials. Structures of the synthesized compounds were characterized by spectroscopic data and CHN analyses. The antimicrobial studies of the synthesized compounds have shown promising activities towards the fungal strains viz., A niger and A. sereus and moderate activities of towards the bacterial strains viz., E.coli and B.subtilis.
\end{abstract}

Keywords 2-pyrazoline. Quinoline. Acetyl sydnone. Antimicrobial Studies

\section{Introduction}

Pyrazolines and related five membered heterocycles have attracted the attention of researchers in recent years ${ }^{1-4}$. Several pyrazoline derivatives have been found to possess promising biological activities, which has stimulated research activity in this field. Their prominent effects are e.g., antimicrobial ${ }^{5}$ central nervous system ${ }^{6}$ and immunosuppressive ${ }^{7}$ activities. After the pioneering work of Fischer and Knövenagel in the late nineteenth century $^{8}$ the reaction of $\alpha, \beta$-unsaturated aldehydes and ketones with hydrazines became one of the most popular methods for the preparation of 2-pyrazolines ${ }^{9-36}$.

In continuation of our work with pharmacologically active compounds we report herein a novel route of synthesis of 2-pyrazoline using the two biologically active moieties of quinoline and acetyl sydnone. It was interesting in knowing the varying biological behavior of the synthesized compounds. An attempt has been carried in evaluation of the synthesized compounds towards the structural elucidation and antimicrobial activities.

\section{Experimental}

Melting points were determined on an Electro-thermal AZ 9000 3MK4 apparatus and were uncorrected. The thin layer chromatography (TLC, $\mathrm{Rf}$ values) was performed on $\mathrm{Al}_{2} \mathrm{O}_{3} 60$ plates F254 or silica gel plates (Merck, $0.2 \mathrm{~mm}$ thick) using mobile phase benzene/ethanol2:0.5, respectively, benzene/ethanol-4:2, and visualization was effected with ultraviolet light. IR spectra were recorded on a Specord 71 IR spectrophotometer as potassium bromide discs. All NMR spectra were recorded on a Bruker Avance DRX 250 spectrometer operating at 
250.13 MHz for ${ }^{1} \mathrm{H}$ NMR. Chemical shifts were expressed relative to tetramethylsilane (TMS) and were reported as $\mathrm{d}(\mathrm{ppm})$. The measurements were carried out at ambient temperature $(300 \mathrm{~K})$. The microanalyses for $\mathrm{C}, \mathrm{H}, \mathrm{N}$ and $\mathrm{S}$ were performed on PerkinElmer elemental analyzer.

General procedure for the synthesis (Compounds $\mathbf{1 a - 1 h}$ )

Compounds 1a-1h (Scheme 1) were prepared as follows: $\mathrm{m} / \mathrm{p}$-acetyl phenyl sydnone $(2.0 \mathrm{~g}$, $0.01 \mathrm{~mol})$ was suspended in sodium hydroxide solution $(0.5 \mathrm{~g})$ in water and ethanol $(5 \mathrm{~mL})$ and 2-mercaptoquinoline-3-carbaldehyde $(1.89 \mathrm{~g}, 0.01 \mathrm{~mol})$ was added to it. The reaction mixture was stirred for 30 minutes at room temperature. The precipitate formed was immediately collected and washed thoroughly with water. The solid obtained was dried and crystallized using DMF. The same procedure is used for the synthesis of 3-[3'-(2"chloroquinoline-1"-oxo-2"-propene-1"-yl)] phenylsydnone and other derivatives (Table 1 and 2).

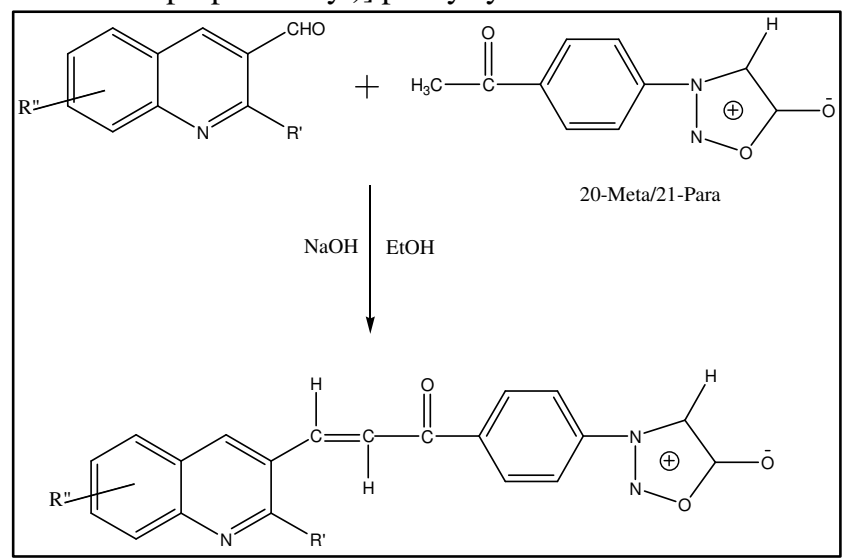

la $R^{\prime}=C l, R^{\prime \prime}=H, 1 b R^{\prime}=S H, R^{\prime \prime}=H, l c R^{\prime}=C l, R^{\prime \prime}=O_{3} H_{3} l d R^{\prime}=S H, R^{\prime \prime}=O C H_{3}$, le $R^{\prime}=C l, R "=O H$, If $R^{\prime}=\mathrm{SH}, \mathrm{R}^{\prime \prime}=\mathrm{OH}, \lg \mathrm{R}^{\prime}=\mathrm{Cl}, \mathrm{R}^{\prime \prime}=\mathrm{NO}_{2}, \mathrm{lh} \mathrm{R}^{\prime}=\mathrm{SH}, \mathrm{R}^{\prime \prime}=\mathrm{NO}_{2}$

Scheme 1. Synthesis of compounds $1 \mathbf{a}-\mathbf{1 h}$

Table 1. Physical properties of compounds $\mathbf{1 a}-\mathbf{1 h}$ (Para isomer)

\begin{tabular}{|c|c|c|c|c|c|c|c|c|}
\hline \multirow[t]{2}{*}{$\begin{array}{l}\text { Compound } \\
\text { Code }\end{array}$} & \multirow[t]{2}{*}{$\mathrm{R}^{\prime}$} & \multirow[t]{2}{*}{$\mathrm{R}^{\prime \prime}$} & \multirow[t]{2}{*}{${ }^{\mathrm{M} . \mathrm{P}}$} & \multirow[t]{2}{*}{$\begin{array}{c}\text { Yield } \\
\%\end{array}$} & \multirow[t]{2}{*}{ Mol. Form } & \multicolumn{3}{|c|}{$\begin{array}{l}\text { Elemental analysis \% } \\
\text { Found (Calc) }\end{array}$} \\
\hline & & & & & & $\mathrm{C}$ & $\mathrm{H}$ & $\mathrm{N}$ \\
\hline $1 \mathrm{a}$ & $\mathrm{Cl}$ & $\mathrm{H}$ & $214-15$ & 88 & $\mathrm{C}_{20} \mathrm{H}_{12} \mathrm{O}_{3} \mathrm{~N}_{3} \mathrm{Cl}$ & $\begin{array}{c}63.58 \\
(63.55)\end{array}$ & $\begin{array}{c}3.17 \\
(3.19)\end{array}$ & $\begin{array}{c}11.12 \\
(11.14)\end{array}$ \\
\hline $1 b$ & $\mathrm{SH}$ & $\mathrm{H}$ & $207-08$ & 78 & $\mathrm{C}_{20} \mathrm{H}_{13} \mathrm{O}_{3} \mathrm{~N}_{3} \mathrm{~S}$ & $\begin{array}{c}64.00 \\
(64.02)\end{array}$ & $\begin{array}{c}3.46 \\
(3.45)\end{array}$ & $\begin{array}{l}11.20 \\
(11.21)\end{array}$ \\
\hline $1 \mathrm{c}$ & $\mathrm{Cl}$ & $\mathrm{OCH}_{3}$ & $193-94$ & 66 & $\mathrm{C}_{21} \mathrm{H}_{14} \mathrm{O}_{4} \mathrm{~N}_{3} \mathrm{Cl}$ & $\begin{array}{c}61.84 \\
(61.89)\end{array}$ & $\begin{array}{l}3.43 \\
(3.48)\end{array}$ & $\begin{array}{l}10.30 \\
(10.32)\end{array}$ \\
\hline $1 d$ & $\mathrm{SH}$ & $\mathrm{OCH}_{3}$ & $211-12$ & 69 & $\mathrm{C}_{21} \mathrm{H}_{15} \mathrm{O}_{4} \mathrm{~N}_{3} \mathrm{~S}$ & $\begin{array}{c}62.22 \\
(62.24)\end{array}$ & $\begin{array}{c}3.70 \\
(3.71)\end{array}$ & $\begin{array}{c}10.37 \\
(10.38)\end{array}$ \\
\hline $1 \mathrm{e}$ & $\mathrm{Cl}$ & $\mathrm{OH}$ & 201-02 & 71 & $\mathrm{C}_{20} \mathrm{H}_{12} \mathrm{O}_{4} \mathrm{~N}_{3} \mathrm{Cl}$ & $\begin{array}{c}60.99 \\
(59.98)\end{array}$ & $\begin{array}{c}3.04 \\
(3.06)\end{array}$ & $\begin{array}{l}10.67 \\
(10.68)\end{array}$ \\
\hline $1 f$ & $\mathrm{SH}$ & $\mathrm{OH}$ & $198-99$ & 82 & $\mathrm{C}_{20} \mathrm{H}_{13} \mathrm{O}_{4} \mathrm{~N}_{3} \mathrm{~S}$ & $\begin{array}{l}61.38 \\
(61.40)\end{array}$ & $\begin{array}{c}3.32 \\
(3.33)\end{array}$ & $\begin{array}{c}10.74 \\
(10.75)\end{array}$ \\
\hline $1 \mathrm{~g}$ & $\mathrm{Cl}$ & $\mathrm{NO}_{2}$ & $215-16$ & 68 & $\mathrm{C}_{20} \mathrm{H}_{11} \mathrm{O}_{5} \mathrm{~N}_{4} \mathrm{Cl}$ & $\begin{array}{c}56.81 \\
(56.84)\end{array}$ & $\begin{array}{l}2.60 \\
(2.63)\end{array}$ & $\begin{array}{c}13.25 \\
(13.27)\end{array}$ \\
\hline $1 \mathrm{~h}$ & $\mathrm{SH}$ & $\mathrm{NO}_{2}$ & $222-23$ & 77 & $\mathrm{C}_{20} \mathrm{H}_{12} \mathrm{O}_{4} \mathrm{~N}_{4} \mathrm{~S}$ & $\begin{array}{c}57.14 \\
(57.16)\end{array}$ & $\begin{array}{l}2.85 \\
(2.87)\end{array}$ & $\begin{array}{c}13.33 \\
(13.35)\end{array}$ \\
\hline
\end{tabular}


Table 2 Physical Properties of compounds 1a - 1h (Meta isomer)

\begin{tabular}{|c|c|c|c|c|c|c|c|c|}
\hline \multirow{2}{*}{$\begin{array}{l}\text { Compound } \\
\text { Code }\end{array}$} & \multirow[t]{2}{*}{$\mathrm{R}^{\prime}$} & \multirow[t]{2}{*}{$\mathrm{R}^{\prime \prime}$} & \multirow{2}{*}{$\begin{array}{l}\text { M.P } \\
{ }^{0} \mathrm{C}\end{array}$} & \multirow{2}{*}{$\begin{array}{l}\text { Yield } \\
\%\end{array}$} & \multirow[t]{2}{*}{ Mol. Form } & \multicolumn{3}{|c|}{$\begin{array}{l}\text { Elemental analysis \% } \\
\text { Found (Calc) }\end{array}$} \\
\hline & & & & & & $\mathrm{C}$ & $\mathrm{H}$ & $\mathrm{N}$ \\
\hline $1 \mathbf{a}$ & $\mathrm{Cl}$ & $\mathrm{H}$ & 202-03 & 85 & $\mathrm{C}_{20} \mathrm{H}_{12} \mathrm{O}_{3} \mathrm{~N}_{3} \mathrm{Cl}$ & $\begin{array}{c}63.58 \\
(63.55)\end{array}$ & $\begin{array}{c}3.17 \\
(3.19)\end{array}$ & $\begin{array}{c}11.12 \\
(11.14)\end{array}$ \\
\hline $1 b$ & $\mathrm{SH}$ & $\mathrm{H}$ & 198-99 & 71 & $\mathrm{C}_{20} \mathrm{H}_{13} \mathrm{O}_{3} \mathrm{~N}_{3} \mathrm{~S}$ & $\begin{array}{c}64.00 \\
(64.02)\end{array}$ & $\begin{array}{c}3.46 \\
(3.45)\end{array}$ & $\begin{array}{c}11.20 \\
(11.21)\end{array}$ \\
\hline $1 \mathrm{c}$ & $\mathrm{Cl}$ & $\mathrm{OCH}_{3}$ & 201-02 & 69 & $\mathrm{C}_{21} \mathrm{H}_{14} \mathrm{O}_{4} \mathrm{~N}_{3} \mathrm{Cl}$ & $\begin{array}{c}61.84 \\
(61.89)\end{array}$ & $\begin{array}{c}3.43 \\
(3.48)\end{array}$ & $\begin{array}{l}10.30 \\
(10.32)\end{array}$ \\
\hline $1 d$ & $\mathrm{SH}$ & $\mathrm{OCH}_{3}$ & $223-24$ & 62 & $\mathrm{C}_{21} \mathrm{H}_{15} \mathrm{O}_{4} \mathrm{~N}_{3} \mathrm{~S}$ & $\begin{array}{c}62.22 \\
(62.24)\end{array}$ & $\begin{array}{c}3.70 \\
(3.71)\end{array}$ & $\begin{array}{c}10.37 \\
(10.38)\end{array}$ \\
\hline 1e & $\mathrm{Cl}$ & $\mathrm{OH}$ & $196-97$ & 78 & $\mathrm{C}_{20} \mathrm{H}_{12} \mathrm{O}_{4} \mathrm{~N}_{3} \mathrm{Cl}$ & $\begin{array}{c}60.99 \\
(59.98)\end{array}$ & $\begin{array}{c}3.04 \\
(3.06)\end{array}$ & $\begin{array}{c}10.67 \\
(10.68)\end{array}$ \\
\hline 1f & $\mathrm{SH}$ & $\mathrm{OH}$ & 204-05 & 88 & $\mathrm{C}_{20} \mathrm{H}_{13} \mathrm{O}_{4} \mathrm{~N}_{3} \mathrm{~S}$ & $\begin{array}{l}61.38 \\
(61.40)\end{array}$ & $\begin{array}{c}3.32 \\
(3.33)\end{array}$ & $\begin{array}{c}10.74 \\
(10.75)\end{array}$ \\
\hline $1 \mathrm{~g}$ & $\mathrm{Cl}$ & $\mathrm{NO}_{2}$ & $228-29$ & 62 & $\mathrm{C}_{20} \mathrm{H}_{11} \mathrm{O}_{5} \mathrm{~N}_{4} \mathrm{Cl}$ & $\begin{array}{c}56.81 \\
(56.84)\end{array}$ & $\begin{array}{c}2.60 \\
(2.63)\end{array}$ & $\begin{array}{c}13.25 \\
(13.27)\end{array}$ \\
\hline $1 \mathrm{~h}$ & $\mathrm{SH}$ & $\mathrm{NO}_{2}$ & 206-07 & 77 & $\mathrm{C}_{20} \mathrm{H}_{12} \mathrm{O}_{4} \mathrm{~N}_{4} \mathrm{~S}$ & $\begin{array}{c}57.14 \\
(57.16)\end{array}$ & $\begin{array}{c}2.85 \\
(2.87)\end{array}$ & $\begin{array}{c}13.33 \\
(13.35)\end{array}$ \\
\hline
\end{tabular}

3-[3'-(2"-Chloroquinoline-1"-oxo-2"-propene-1"-yl)] phenylsydnone (1a)

Yield 88\%; mp 214-15 ${ }^{0} \mathrm{C}$, re-crystallized with ethanol; IR $(\mathrm{KBr}) \mathrm{cm}^{-1} 3111,1732,1654 .{ }^{1} \mathrm{H}$ NMR (DMSO-d6) 7.3 (s, 1H, C4 proton of sydnone ring), 7.5 (s, 1H, C4 proton of sydnone ring), 7.1-8.4 (m, 9H, Ar-H).

3-[3'-(2"-Mercaptoquinoline-1"-oxo-2"-propene-1"-yl)] phenylsydnone (2a)

Yield 78\%; mp 207-08 ${ }^{0} \mathrm{C}$, re-crystallized with ethanol; IR $(\mathrm{KBr}) \mathrm{cm}^{-1} 3113,1740,1660 .{ }^{1} \mathrm{H}$ NMR (DMSO-d6) 7.3 (s, 1H, C4 proton of sydnone ring), 3.1 (s, 1H, SH Proton), 7.4 (s, 1H, $\mathrm{C} 4$ proton of sydnone ring), 7.2-8.3 (m, $9 \mathrm{H}, \mathrm{Ar}-\mathrm{H})$.

[3'-(2"-Chloro-7"-methoxyquinoline-1"-oxo-2"-propene-1"-yl)] phenylsydnone (3a) Yield 66\%; mp 193-94 ${ }^{0} \mathrm{C}$, re-crystallized with ethanol; IR $(\mathrm{KBr}) \mathrm{cm}^{-1} 3112,1741,1646 .{ }^{1} \mathrm{H}$ NMR (DMSO-d6) 7.3 (s, 1H, C4 proton of sydnone ring), $3.74\left(3 \mathrm{H}, s,-\mathrm{OCH}_{3}\right), 7.2(\mathrm{~s}, 1 \mathrm{H}$, $\mathrm{C} 4$ proton of sydnone ring), 7.2-8.4 (m, $9 \mathrm{H}, \mathrm{Ar}-\mathrm{H})$.

3-[3'-(2"-Mercapto-7"-methoxyquinoline-1"-oxo-2"-propene-1"-yl)] phenylsydnone (4a) Yield 69\%; mp 211-12 ${ }^{0} \mathrm{C}$, re-crystallized with ethanol; IR $(\mathrm{KBr}) \mathrm{cm}^{-1} 3115,1744,1651 .{ }^{1} \mathrm{H}$ NMR (DMSO-d6) 7.3 (s, 1H, C4 proton of sydnone ring), 2.8 (s, 1H, SH Proton), 3.75 (3H, $\left.s,-\mathrm{OCH}_{3}\right), 7.1$ (s, $1 \mathrm{H}, \mathrm{C} 4$ proton of sydnone ring), 7.1-8.2 (m, $\left.9 \mathrm{H}, \mathrm{Ar}-\mathrm{H}\right)$.

3-[3'-(2"-Chloro-7"-hydroxyquinoline-1"-oxo-2"-propene-1"-yl)] phenylsydnone (5a) Yield 71\%; mp 201-02 ${ }^{\circ} \mathrm{C}$, re-crystallized with ethanol; IR $(\mathrm{KBr}) \mathrm{cm}^{-1} 3125,1742,1661 .{ }^{1} \mathrm{H}$ NMR (DMSO-d6) 7.2 (s, 1H, C4 proton of sydnone ring), 7.3 (s, 1H, C4 proton of sydnone ring), 7.0-8.3 (m, 9H, Ar-H), $13.3\left(1 \mathrm{H}, \mathrm{s}, \mathrm{OH}, \mathrm{D}_{2} \mathrm{O}\right.$ exchanged). 
3-[3'-(2"-Mercapto-7"-hydroxyquinoline-1"-oxo-2"-propene-1"-yl)] phenylsydnone (6a) Yield 82\%; mp 198-99 ${ }^{0} \mathrm{C}$, re-crystallized with ethanol; IR $(\mathrm{KBr}) \mathrm{cm}^{-1} 3122,1735,1659 .{ }^{1} \mathrm{H}$ NMR (DMSO-d6) 7.5 (s, 1H, C4 proton of sydnone ring), 3.0 (s, 1H, SH Proton), 7.2 (s, 1H, $\mathrm{C} 4$ proton of sydnone ring), 7.2-8.5 (m, $9 \mathrm{H}, \mathrm{Ar}-\mathrm{H}), 13.5\left(1 \mathrm{H}, \mathrm{s}, \mathrm{OH}, \mathrm{D}_{2} \mathrm{O}\right.$ exchanged).

3-[3'-(2"-Chloro-7"-nitroquinoline-1"-oxo-2"-propene-1"-yl)] phenylsydnone (7a)

Yield $68 \%$; mp 215-16 ${ }^{\circ} \mathrm{C}$, re-crystallized with ethanol; IR $(\mathrm{KBr}) \mathrm{cm}^{-1} 3108,1732,1660 .{ }^{1} \mathrm{H}$ NMR (DMSO-d6) 7.4 (s, 1H, C4 proton of sydnone ring), 7.3 (s, 1H, C4 proton of sydnone ring), 7.1-8.2 (m, 9H, Ar-H).

3-[3'-(2"-Mercapto-7"-nitroquinoline-1"-oxo-2"-propene-1"-yl)] phenylsydnone (8a) Yield 77\%; mp 222-23 ${ }^{0} \mathrm{C}$, re-crystallized with ethanol; IR $(\mathrm{KBr}) \mathrm{cm}^{-1} 3110,1730,1658 .{ }^{1} \mathrm{H}$ NMR (DMSO-d6) 7.2 (s, 1H, C4 proton of sydnone ring), 2.9 (s, 1H, SH Proton), 7.0 (s, 1H, $\mathrm{C} 4$ proton of sydnone ring), 7.2-8.4 (m, $9 \mathrm{H}, \mathrm{Ar}-\mathrm{H})$.

General procedure for the synthesis (Compounds $2 \mathbf{a}-2 \mathrm{~h}$ )

Compounds $\mathbf{2 a - 2 h}$ (Scheme 2) was prepared by a mixture of 3-aryl-[4'-(3"-quinoline-1"oxo-2"-propen-1"-yl)]-sydnone (1.84 g, $0.005 \mathrm{~mol})$ and phenyl hydrazine hydrate $(0.5 \mathrm{~mL}$, $0.005 \mathrm{~mol})$ in acetic acid $(15 \mathrm{~mL})$ was refluxed on water bath for $3 \mathrm{~h}$. Progress of the reaction was monitored by TLC.

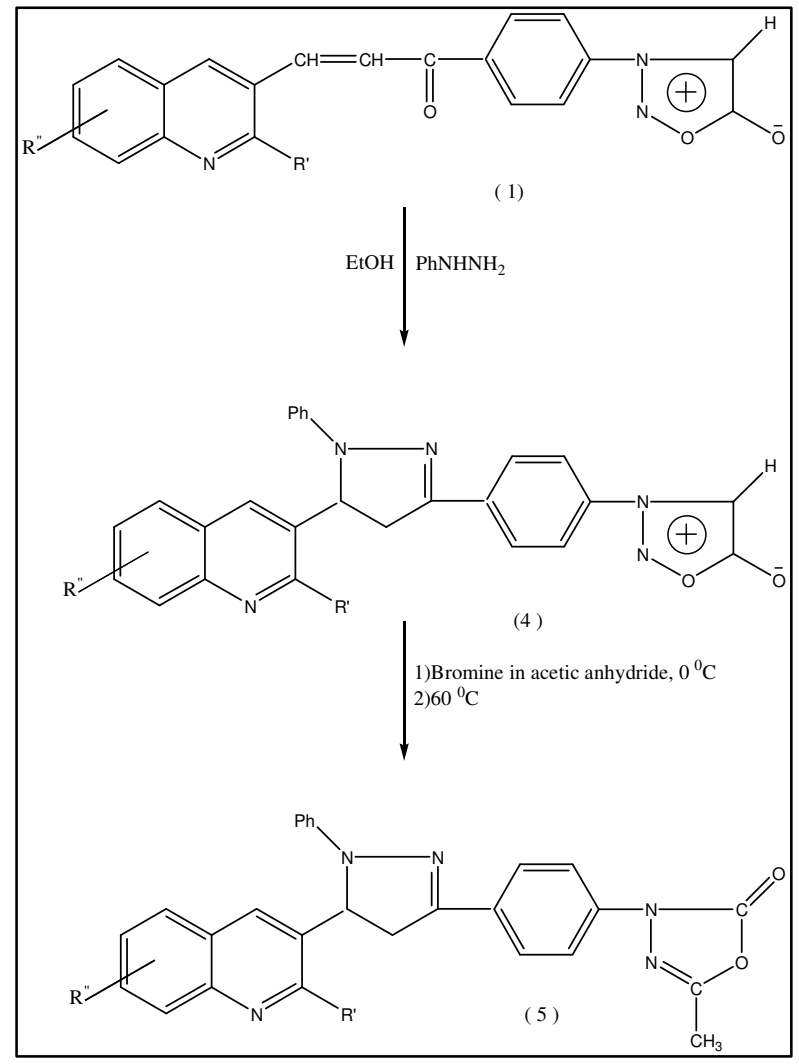

Scheme 2 Synthesis of Pyrazoles $\mathbf{2 a}-\mathbf{2 h}$ 
After completion of the reaction, the resultant clear solution was poured into ice-cold water. The precipitate formed was filtered and dried. The solid on crystallization in ethanol afforded the light yellow crystalline compound 3-[4'-(1"-acyl-5"- quinoline-2"-pyrazolin3"-yl)phenyl]sydnone $484 \%$ (2.73 g). Compound 4 (2.61g, $0.005 \mathrm{~mol})$ was suspended in acetic anhydride $(10 \mathrm{~mL})$ and to this a mixture of bromine $(0.3 \mathrm{~mL}, 0.055 \mathrm{~mol})$ in acetic anhydride $(5 \mathrm{~mL})$ was added with constant stirring at $0{ }^{\circ} \mathrm{C}$. After completion of addition, the stirring was continued for another 30 minutes. Then the reaction mixture was warmed on a water bath at $60{ }^{\circ} \mathrm{C}$ until evolution of carbon dioxide ceases. Then the reaction mixture was then poured into water, solid separated was filtered and dried. The separated solid on crystallization ethanol afforded $\mathbf{5}$ as light yellow crystalline compound in $68 \%$ yield (Scheme 2 \& Table 3).

Table 3. Physical properties of pyrazoles $\mathbf{2 a}$ to $\mathbf{2 h}$ (Para isomer only)

\begin{tabular}{|c|c|c|c|c|c|c|c|c|}
\hline \multirow{2}{*}{$\begin{array}{l}\text { Compound } \\
\text { Code }\end{array}$} & \multirow[t]{2}{*}{$\mathrm{R}^{\prime}$} & \multirow[t]{2}{*}{$\mathrm{R}^{\prime \prime}$} & \multirow{2}{*}{$\stackrel{\text { M.P }}{{ }^{0} \mathrm{C}}$} & \multirow{2}{*}{$\begin{array}{l}\text { Yield } \\
\%\end{array}$} & \multirow[t]{2}{*}{ Mol. Form } & \multicolumn{3}{|c|}{$\begin{array}{l}\text { Elemental analysis \% } \\
\text { Found (Calc) }\end{array}$} \\
\hline & & & & & & $\mathrm{C}$ & $\mathrm{H}$ & $\mathrm{N}$ \\
\hline $2 \mathbf{a}$ & $\mathrm{Cl}$ & $\mathrm{H}$ & $172-73$ & 72 & $\mathrm{C}_{27} \mathrm{H}_{20} \mathrm{O}_{2} \mathrm{~N}_{5} \mathrm{Cl}$ & $\begin{array}{c}67.29 \\
(67.30)\end{array}$ & $\begin{array}{c}4.15 \\
(4.16)\end{array}$ & $\begin{array}{c}14.53 \\
(14.52)\end{array}$ \\
\hline $2 b$ & $\mathrm{SH}$ & $\mathrm{H}$ & $187-88$ & 73 & $\mathrm{C}_{27} \mathrm{H}_{21} \mathrm{O}_{2} \mathrm{~N}_{5} \mathrm{~S}$ & $\begin{array}{c}67.64 \\
(67.65)\end{array}$ & $\begin{array}{c}4.38 \\
(4.47)\end{array}$ & $\begin{array}{c}14.61 \\
(14.62)\end{array}$ \\
\hline $2 c$ & $\mathrm{Cl}$ & $\mathrm{OCH}_{3}$ & $168-69$ & 85 & $\mathrm{C}_{28} \mathrm{H}_{22} \mathrm{O}_{3} \mathrm{~N}_{5} \mathrm{Cl}$ & $\begin{array}{c}65.69 \\
(65.70)\end{array}$ & $\begin{array}{c}4.30 \\
(4.31)\end{array}$ & $\begin{array}{c}13.68 \\
(13.67)\end{array}$ \\
\hline 2d & $\mathrm{SH}$ & $\mathrm{OCH}_{3}$ & 211-12 & 86 & $\mathrm{C}_{28} \mathrm{H}_{23} \mathrm{O}_{3} \mathrm{~N}_{5} \mathrm{~S}$ & $\begin{array}{c}66.01 \\
(66.02)\end{array}$ & $\begin{array}{c}4.51 \\
(4.52)\end{array}$ & $\begin{array}{c}13.75 \\
(13.76)\end{array}$ \\
\hline $2 e$ & $\mathrm{Cl}$ & $\mathrm{OH}$ & 198-99 & 74 & $\mathrm{C}_{27} \mathrm{H}_{20} \mathrm{O}_{3} \mathrm{~N}_{5} \mathrm{Cl}$ & $\begin{array}{c}65.13 \\
(65.14)\end{array}$ & $\begin{array}{c}4.02 \\
(4.03)\end{array}$ & $\begin{array}{c}14.07 \\
(14.06)\end{array}$ \\
\hline $2 f$ & $\mathrm{SH}$ & $\mathrm{OH}$ & 193-94 & 77 & $\mathrm{C}_{27} \mathrm{H}_{21} \mathrm{O}_{3} \mathrm{~N}_{5} \mathrm{~S}$ & $\begin{array}{c}65.45 \\
(65.46)\end{array}$ & $\begin{array}{c}4.24 \\
(4.25)\end{array}$ & $\begin{array}{c}14.14 \\
(14.13)\end{array}$ \\
\hline $2 \mathrm{~g}$ & $\mathrm{Cl}$ & $\mathrm{NO}_{2}$ & $212-13$ & 68 & $\mathrm{C}_{27} \mathrm{H}_{19} \mathrm{O}_{4} \mathrm{~N}_{6} \mathrm{Cl}$ & $\begin{array}{c}61.54 \\
(61.55)\end{array}$ & $\begin{array}{c}3.60 \\
(3.61)\end{array}$ & $\begin{array}{c}15.95 \\
(15.96)\end{array}$ \\
\hline $2 \mathrm{~h}$ & $\mathrm{SH}$ & $\mathrm{NO}_{2}$ & $185-86$ & 70 & $\mathrm{C}_{27} \mathrm{H}_{20} \mathrm{O}_{4} \mathrm{~N}_{6} \mathrm{~S}$ & $\begin{array}{r}61.83 \\
(61.84) \\
\end{array}$ & $\begin{array}{c}3.81 \\
(3.83) \\
\end{array}$ & $\begin{array}{c}16.03 \\
(16.02) \\
\end{array}$ \\
\hline
\end{tabular}

3-\{-[5-(2-Chloroquinolin-3-yl)-1-phenyl-1H-pyrazol-3-yl] phenyl\}-5-methyl1,3,4-oxadiazol-2(3H)-one (2a)

Yield $72 \%$; mp $172-73{ }^{\circ} \mathrm{C}$, re-crystallized with ethanol; IR $(\mathrm{KBr}), v_{\max } / \mathrm{cm}^{-1}$ : 1776(lactone $\mathrm{C}=\mathrm{O}), 1690 ;{ }^{1} \mathrm{H}$ NMR $\left(\mathrm{CDCl}_{3}\right), \delta: 2.31\left(3 \mathrm{H}, \mathrm{s}, \mathrm{C}-5 \mathrm{CH}_{3}\right), 8.62(\mathrm{dd}, 1 \mathrm{H}, \mathrm{H}-5), 7.82(\mathrm{~d}, \mathrm{~J}=8.1$ $\mathrm{Hz}, 1 \mathrm{H}, \mathrm{H}-6), 7.65-7.54$ (m, 2H, H-7, H-8), 7.45-7.15 (br-m, 9H, Ar-H), 5.20 (dd, 1H, CHx), $4.2\left(\mathrm{dd}, 1 \mathrm{H}, \mathrm{H}_{\mathrm{A}}\right), 3.61\left(\mathrm{dd}, 1 \mathrm{H}, \mathrm{H}_{\mathrm{B}}\right)$.

3-\{-[5-(2-Mercaptoquinolin-3-yl)-1-phenyl-1H-pyrazol-3-yl] phenyl\}-5-methyl-1,3, 4-oxadiazol-2(3H)-one (2b)

Yield 73\%; mp 187-88 ${ }^{0} \mathrm{C}$, re-crystallized with ethanol; IR (KBr), $v_{\max } / \mathrm{cm}^{-1}$ : 1775 (lactone $\mathrm{C}=\mathrm{O}), 1678 ;{ }^{1} \mathrm{H} \mathrm{NMR}\left(\mathrm{CDCl}_{3}\right), \delta: 2.0\left(3 \mathrm{H}, \mathrm{s}, \mathrm{C}-5 \mathrm{CH}_{3}\right), 8.62(\mathrm{dd}, 1 \mathrm{H}, \mathrm{H}-5), 7.84(\mathrm{~d}, 1 \mathrm{H}, \mathrm{H}-$ 6), 7.62-7.53 (m, 2H, H-7, H-8), 3.0 (s, 1H, SH Proton), 7.41-7.12 (br-m, 9H, Ar-H), 5.20 (dd, 1H, CHx), $4.2\left(\mathrm{dd}, 1 \mathrm{H}, \mathrm{H}_{\mathrm{A}}\right), 3.61\left(\mathrm{dd}, 1 \mathrm{H}, \mathrm{H}_{\mathrm{B}}\right)$. 
3-\{-[5-(2-Chlor-7-methoxyoquinolin-3-yl)-1-phenyl-1H-pyrazol-3-yl] phenyl\}5-methyl-1,3,4-oxadiazol-2(3H)-one (2c)

Yield 85\%; mp 168-69 ${ }^{0} \mathrm{C}$, re-crystallized with ethanol; IR $(\mathrm{KBr}), v_{\max } / \mathrm{cm}^{-1}: 1772$ (lactone $\mathrm{C}=\mathrm{O}), 1667 ;{ }^{1} \mathrm{H}$ NMR $\left(\mathrm{CDCl}_{3}\right), \delta: 1.9\left(3 \mathrm{H}, \mathrm{s}, \mathrm{C}-5 \mathrm{CH}_{3}\right), 8.62(\mathrm{dd}, 1 \mathrm{H}, \mathrm{H}-5), 7.80(\mathrm{~d}, \mathrm{~J}=8.1$ Hz, 1H, H-6), 7.60-7.52 (m, 2H, H-7, H-8), 7.43-7.10 (br-m, 9H, Ar-H), 5.18 (dd, 1H, CHx), $4.30\left(\mathrm{dd}, \mathrm{J}=12.3,19.2 \mathrm{~Hz}, 1 \mathrm{H}, \mathrm{H}_{\mathrm{A}}\right), 3.76\left(\mathrm{~s}, 3 \mathrm{H}, \mathrm{OCH}_{3}\right), 3.61\left(\mathrm{dd}, \mathrm{J}=7.2,19.2 \mathrm{~Hz}, 1 \mathrm{H}, \mathrm{H}_{\mathrm{B}}\right)$.

3-\{-[5-(2-Mercapto-7-methoxyoquinolin-3-yl)-1-phenyl-1H-pyrazol-3-yl]phenyl\}-5methyl-1,3,4-oxadiazol-2(3H)-one (2d)

Yield 86\%; mp 211-12 ${ }^{\circ} \mathrm{C}$, re-crystallized with ethanol; IR $(\mathrm{KBr}), v_{\max } / \mathrm{cm}^{-1}: 1777$ (lactone $\mathrm{C}=\mathrm{O}), 1677 ;{ }^{1} \mathrm{H}$ NMR $\left(\mathrm{CDCl}_{3}\right), \delta: 1.7\left(3 \mathrm{H}, \mathrm{s}, \mathrm{C}-5 \mathrm{CH}_{3}\right), 8.62(\mathrm{dd}, \mathrm{J}=7.8,1.2 \mathrm{~Hz}, 1 \mathrm{H}, \mathrm{H}-5)$, 7.80 (d, 1H, H-6), 7.63-7.56 (m, 2H, H-7, H-8), 3.4 (s, 1H, SH Proton), 7.53-7.16 (br-m, $9 \mathrm{H}, \mathrm{Ar}-\mathrm{H}), 5.18(\mathrm{dd}, \mathrm{J}=7.2,12.3 \mathrm{~Hz}, 1 \mathrm{H}, \mathrm{CHx}), 4.29\left(\mathrm{dd}, 1 \mathrm{H}, \mathrm{H}_{\mathrm{A}}\right), 3.71\left(\mathrm{~s}, 3 \mathrm{H}, \mathrm{OCH}_{3}\right)$, $3.69\left(\mathrm{dd}, \mathrm{J}=7.2,19.2 \mathrm{~Hz}, 1 \mathrm{H}, \mathrm{H}_{\mathrm{B}}\right)$.

3-\{-[5-(2-Chloro-7-hydroxyoquinolin-3-yl)-1-phenyl-1H-pyrazol-3-yl]phenyl\}-5methyl-1,3,4-oxadiazol-2(3H)-one (2e)

Yield 74\%; mp 198-99 ${ }^{0} \mathrm{C}$, re-crystallized with ethanol; IR (KBr), $v_{\max } / \mathrm{cm}^{-1}: 1772$ (lactone $\mathrm{C}=\mathrm{O}), 1675 ;{ }^{1} \mathrm{H}$ NMR $\left(\mathrm{CDCl}_{3}\right), \delta: 2.2\left(3 \mathrm{H}, \mathrm{s}, \mathrm{C}-5 \mathrm{CH}_{3}\right), 8.66$ (dd, $\left.1 \mathrm{H}, \mathrm{H}-5\right), 7.85$ (d, J = 8.1 $\mathrm{Hz}, 1 \mathrm{H}, \mathrm{H}-6), 7.68-7.56$ (m, 2H, H-7, H-8), 7.55-7.11 (br-m, 9H, Ar-H), 5.10 (dd, 1H, CHx), $4.29\left(\mathrm{dd},, 1 \mathrm{H}, \mathrm{H}_{\mathrm{A}}\right), 3.69\left(\mathrm{dd}, 1 \mathrm{H}, \mathrm{H}_{\mathrm{B}}\right)$.

3-\{-[5-(2-Mercapto-7-hydroxyoquinolin-3-yl)-1-phenyl-1H-pyrazol-3-yl]phenyl\}-5methyl-1,3,4-oxadiazol-2(3H)-one (2f)

Yield 74\%; mp 198-99 ${ }^{0} \mathrm{C}$, re-crystallized with ethanol; IR (KBr), $v_{\max } / \mathrm{cm}^{-1}: 1773$ (lactone $\mathrm{C}=\mathrm{O}), 1671 ;{ }^{1} \mathrm{H}$ NMR $\left(\mathrm{CDCl}_{3}\right), \delta: 2.0\left(3 \mathrm{H}, \mathrm{s}, \mathrm{C}-5 \mathrm{CH}_{3}\right), 8.63$ (dd, $\left.1 \mathrm{H}, \mathrm{H}-5\right), 7.85(\mathrm{~d}, 1 \mathrm{H}, \mathrm{H}-$ 6), 7.61-7.54 (m, 2H, H-7, H-8), 3.2 (s, 1H, SH Proton), 7.52-7.10 (br-m, 9H, Ar-H), 5.15 (dd, 1H, CHx), 4.20(dd, , 1H, $\left.\mathrm{H}_{\mathrm{A}}\right), 3.66\left(\mathrm{dd}, 1 \mathrm{H}, \mathrm{H}_{\mathrm{B}}\right)$.

3-\{-[5-(2-Chloro-7-nitroquinolin-3-yl)-1-phenyl-1H-pyrazol-3-yl]phenyl\}-5-methyl1,3,4-oxadiazol-2(3H)-one (2g)

Yield 68\%; mp 212-13 ${ }^{0} \mathrm{C}$, re-crystallized with ethanol; IR (KBr), $v_{\max } / \mathrm{cm}^{-1}$ : 1771(lactone $\mathrm{C}=\mathrm{O}), 1678 ;{ }^{1} \mathrm{H}$ NMR $\left(\mathrm{CDCl}_{3}\right), \delta: 1.7\left(3 \mathrm{H}, \mathrm{s}, \mathrm{C}-5 \mathrm{CH}_{3}\right), 8.42(\mathrm{dd}, \mathrm{J}=7.8,1.2 \mathrm{~Hz}, 1 \mathrm{H}, \mathrm{H}-5)$, 7.94 (d, J = 8.1 Hz, 1H, H-6), 7.68-7.54 (m, 2H, H-7, H-8), 7.52-7.10 (br-m, 9H, Ar-H), 6.12 $(\mathrm{dd}, \mathrm{J}=7.5,12.6 \mathrm{~Hz}, 1 \mathrm{H}, \mathrm{CHx}), 4.32\left(\mathrm{dd}, 1 \mathrm{H}, \mathrm{H}_{\mathrm{A}}\right), 3.68\left(\mathrm{dd}, \mathrm{J}=7.5,19.2 \mathrm{~Hz}, 1 \mathrm{H}, \mathrm{H}_{\mathrm{B}}\right)$.

3-\{-[5-(2-Mercapto-7-nitroquinolin-3-yl)-1-phenyl-1H-pyrazol-3-yl]phenyl\}-5-methyl -1,3,4-oxadiazol-2(3H)-one (2h)

Yield 70\%; mp 185-86 ${ }^{0} \mathrm{C}$, re-crystallized with ethanol; IR (KBr), $v_{\max } / \mathrm{cm}^{-1}$ : 1771(lactone $\mathrm{C}=\mathrm{O}), 1678 ;{ }^{1} \mathrm{H}$ NMR $\left(\mathrm{CDCl}_{3}\right), \delta: 2.5\left(3 \mathrm{H}, \mathrm{s}, \mathrm{C}-5 \mathrm{CH}_{3}\right), 8.14(\mathrm{dd}, \mathrm{J}=8.1,1.5 \mathrm{~Hz}, 1 \mathrm{H}, \mathrm{H}-5)$, $7.94(\mathrm{~d}, \mathrm{~J}=8.1 \mathrm{~Hz}, 1 \mathrm{H}, \mathrm{H}-6), 7.49$ (t, J = 7.2 Hz, 1H, H-7), 3.1 (s, 1H, SH Proton), 7.52$7.10(\mathrm{br}-\mathrm{m}, 9 \mathrm{H}, \mathrm{Ar}-\mathrm{H}), 6.12(\mathrm{dd}, \mathrm{J}=7.5,12.6 \mathrm{~Hz}, 1 \mathrm{H}, \mathrm{CHx}), 4.32\left(\mathrm{dd},, 1 \mathrm{H}, \mathrm{H}_{\mathrm{A}}\right), 3.68(\mathrm{dd}, \mathrm{J}$ $=7.5,19.2 \mathrm{~Hz}, 1 \mathrm{H}, \mathrm{H}_{\mathrm{B}}$ ).

All the newly synthesized compounds were screened for their antimicrobial activity by cup plate method at $100 \mu \mathrm{g} / \mathrm{mL}$ concentration in DMF against the Bacterial strains viz., E. coli \& $B$. subtilis and also against Fungal strains viz., A. niger and A. sereus. Norfloxacin for bacteria and Griseofulvin as the reference drugs respectively. All these compounds were less active against the bacterial strains, but some of them showed selective fungal inhibitory activity (Table 4). 
Table 4. Antimicrobial activities of synthesized pyrazoles $\mathbf{2 a}$ to $\mathbf{2 h}$

\begin{tabular}{ccccc}
\hline Compound & \multicolumn{2}{c}{ Antibacterial } & \multicolumn{2}{c}{ Antifungal } \\
Code & E.coli & B. subtilis & A. niger & C. albicans \\
\hline $\mathbf{2 a}$ & 12 & 11 & 18 & 17 \\
$\mathbf{2 b}$ & 13 & 14 & 22 & 21 \\
$\mathbf{2 c}$ & 14 & 15 & 21 & 19 \\
$\mathbf{2 d}$ & 15 & 14 & 20 & 21 \\
$\mathbf{2 e}$ & 13 & 14 & 21 & 20 \\
$\mathbf{2 f}$ & 13 & 13 & 18 & 17 \\
$\mathbf{2 g}$ & 11 & 10 & 16 & 15 \\
$\mathbf{2 h}$ & 12 & 11 & 17 & 16 \\
Norfloxacin & 22 & 22 & -- & -- \\
Grisiofulvin & -- & -- & 24 & 24 \\
DMF & 04 & 04 & 04 & 04 \\
\hline
\end{tabular}

The synthesized compounds were characterized by various spectral studies. Compounds $\mathbf{2 b}$ - $2 \mathbf{e}$ were found to be more susceptible towards the fungal strains as compared to bacterial strains (Figure1 and 2).

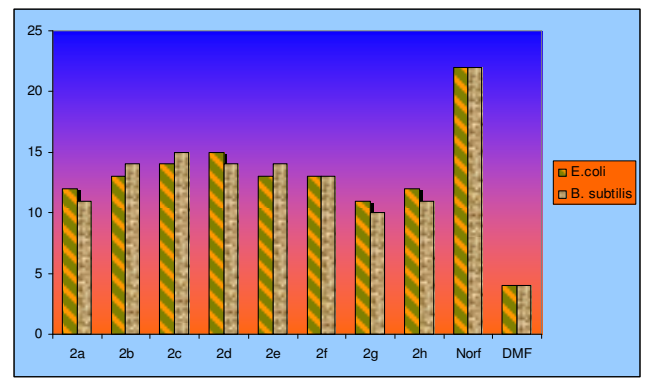

Figure 1. Antibacterial activity of Pyrazoles 2a to $2 \mathbf{h}$

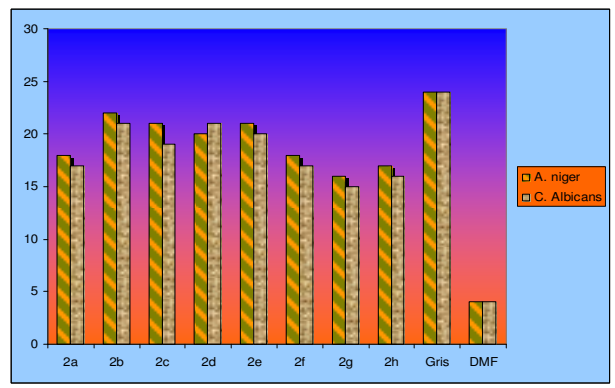

Figure 2. Antifungal activity of Pyrazoles $\mathbf{2 a}$ to $2 \mathbf{h}$

\section{Results and Discussion}

Compound 1, were prepared by acetyl phenyl sydnone was suspended in sodium hydroxide solution in water and ethanol and 2-mercaptoquinoline-3-carbaldehyde was added to it. The reaction mixture was stirred at room temperature (Scheme 1). Further, the compound 1 and phenyl hydrazine hydrate in acetic acid was refluxed on water bath for 3 hours, to give light yellow crystalline compound 3-[4'-(1"'-acyl-5"-quinoline-2"'-pyrazolin-3"'-yl)phenyl] sydnone (4). Further, more the compound (4) was suspended in acetic anhydride and to this a mixture of bromine in acetic anhydride was added with constant stirring at $0{ }^{0} \mathrm{C}$. After completion of addition, the stirring was continued for another 30 minutes to give light yellow crystalline compound (5) (Scheme 2).

The structures of all newly prepared 2-pyrazolines were confirmed by spectroscopic data and elemental analyses. In general, all the compounds exhibited $\mathrm{C}=\mathrm{N}$ stretching vibrations in the range of $1691-1454 \mathrm{~cm}^{-1}$. ${ }^{1} \mathrm{H}$ NMR spectra of these compounds exhibited an $\mathrm{ABX}$ pattern because of the presence of two diastereotopic protons at C-4 and a single proton at $\mathrm{C}-5$ of 2-pyrazoline. Their signals appeared in general as three doublet of doublets in the range of $\delta=6.18-5.17,4.35-4.21$, and 3.72-3.26, each integrating to one proton. A similar ${ }^{1} \mathrm{H}$ NMR pattern in 2-pyrazolines was reported in literature ${ }^{22}$. It is interesting that in 
the ${ }^{1} \mathrm{H}$ NMR spectra, a doublet of doublets integrating to one proton appears in the region of $\delta=8.62-8.16$. This is due to $\mathrm{H}-5$ of the quinolinyl ring system. The large deshielding of this proton was attributed to the anisotropy of $\mathrm{C}=\mathrm{N}$ of the pyrazoline ring. ${ }^{13} \mathrm{C}$ NMR chemical shift values of C-atoms at $\delta=62-54$ (C-5) and 47-42 (C-4) confirmed the 2-pyrazoline character deduced from the ${ }^{1} \mathrm{H}$ NMR data, similar to earlier reported results ${ }^{22}$. The presences of $N$-methyl and $N$-ethyl groups were also confirmed by the ${ }^{1} \mathrm{H}$ NMR spectra.

\section{Acknowledgement}

The authors are thankful to USIC Dharwad for providing the spectral studies. Authorities of BioGenics Hubli for providing facilities in carrying out the biological studies of the compounds.

\section{References}

1. Wiley RH (Ed.), The Chemistry of Heterocyclic Compounds; Weissberger A (Ed.) Interscience Publishers: New York 1967, 22, 180.

2. Elguero J, In Comprehensive Heterocyclic Chemistry II. Katritzky A R, Rees C W, Scriven EF (Eds.) Pergamon Press: Oxford. 1996, 3, 1.

3. Lévai A, Khim Geterotsikl Soedin., 1997, 747,

4. Lévai A J, Heterocycl Chem., 2002, 39,1.

5. Ramalingham K, Thyvekikakath GX, Berlin K D, Chesnut R W, Brown R A, Durham N N, Ealick A E and van der Helm D, J Med Chem., 1977, 20, 847.

6. Brown R E and Jr Shavrel J, U S Patent 1972, 3, 624, 102, Chem Abstr., 1972 76, 59618.

7. Lombardino J G and Otterness I G, J Med Chem., 1981, 24, 830.

8. Fischer E and Knövenagel O, Ann Chem., 1887, 239,194.

9. Raiford L C and Peterson W J, J Org Chem., 1937, 1,544.

10. Raiford L C and Gundy G V, J Org Chem., 1938, 3, 265.

11. Raiford L C and Manley R H, J Org Chem., 1940, 5, 590.

12. Ried W and Dankert G, Chem Ber.. 1957, 90, 2707.

13. Wiley R H, Jarboe C H, Hayes F N, Hansbury E, Nielsen J T, Callahan P X and Sellars M C, J.Org Chem., 1958, 23, 732.

14. Sammour A E A, Tetrahedron, 1964, 20, 1067.

15. Bhatnagar I, George M V, Tetrahedron, 1968, 24, 1293.

16. Aubagnac J L, Elguero J and Jacquier R, Bull Soc Chim Fr., 1969, 3292.

17. Kabli R A, Khalaf A A, Zimaity M T, Khalil A M, Kaddah A M and Al-Rifaie H A, $J$ Indian Chem Soc., 1991, 68, 47.

18. Gokhan N, Ye silada A, Ucar G and Erol K, Arch Pharm Hung., 2003, 336, 362.

19. Sharma T C, Pawar S R and Reddy N J, Acta Chim., 1983, 112, 159.

20. Dhar D N and Raghunathan R, Indian J Chem., 1984, 23B, 1187.

21. Orlov V D, Aziz M A, Mchedlov-Petrosyan N O and Asoka P K D, Khim Geterotsikl Soedin., 1985, 1511.

22. Levai A, Jeko J and Brahmbhatt D I, J Heterocyclic Chem., 2005, 42, 1231.

23. Levai A and Jeko J, J Heterocyclic Chem., 2006, 43, 111.

24. Tóth G, Szöllősy Á, Lóránd T, Kónya T, Szabó D, Földesi A and Lévai A, J Chem Soc Perkin Trans., 1989, 2, 319.

25. Szöllősy Á, Tóth G, Lóránd T, Kónya T, Aradi F and Lévai A, J Chem Soc., Perkin Trans., 1991, 2, 489.

26. Collins L and Franzblau S G, Antimicrob Agents Chemother., 1997, 41, 1004.

27. Bilgin A A, Palaska E, Sunal R and Gümüsel B, Pharmazie, 1994, 49, 67. 
28. Mishriky N, Asaad F M, Ibrahim Y A and Girgis A S, Pharmazie, 1996, 51, 544.

29. Levai A, Heterocycl Commun., 2003, 9, 287.

30. Chimenti F, Bizzarri B, Manna F, Boalsco A, Secci D, Chimenti P, Granese A, Rivanera D, Lilli D, Scaltrito M M and Brenciaglia M I, Bioorg Med Chem Lett., 2005, 15, 603.

31. Dighade S R and Chincholkar M M, Asian J Chem., 2001, 13, 606.

32. Wang P, Onozawa-Komatsuzaki N, Himeda Y, Sugihara H, Arakawa H and Kasuga K, Tetrahedron Lett., 2001, 42, 9199.

33. Manna F, Chimenti F, Bolasco A, Secci D, Bizzarri B, Befani O, Turini P, Mondovi B, Alcaro S and Tafi A, Bioorg Med Chem Lett., 2002, 12, 3629.

34. Lévai A, Patonay T, Silva A M S, Pinto D C G A and Cavaleiro J A S, J Heterocycl Chem., 2002, 39, 751.

35. Lévai A, Heterocycl Commun., 2003, 9, 287.

36. Lévai A, Silva A M S, Pinto D C G A, Cavaleiro J A S, Alkorta I, Elguero J and Jekö J, Eur J Org Chem., 2004, 4672. 